\title{
Review
}

\section{Development of a replication-selective, oncolytic poxvirus for the treatment of human cancers}

\author{
Herbert J Zeh and David L Bartlett
}

\author{
Division of Surgical Oncology, University of Pittsburgh, University of Pittsburgh Cancer Institute, PB02 Cancer \\ Pavilion, Pittsburgh, Pennsylvania 15232, USA.
}

\begin{abstract}
Tumor directed gene therapy for the purpose of destroying cancer cells through replicative "oncolysis" or by intratumoral expression of toxic or immunostimulatory genes requires an efficient, tumor targeted vector. Vectors are limited by inefficient replication in vivo, inefficient tumor targeting, and safety concerns. As a unique approach to addressing these limitations, our laboratory has studied poxviruses as tumor selective replicating vectors. The best in vivo antitumor results achieved to date have been with a mutated WR strain of vaccinia virus. The unique advantage of this strain of vaccinia over other vectors currently being explored for this purpose is the efficiency of in vivo replication. Intradermal injection of $10^{6} \mathrm{pfu}$ of the wild type (non-mutated) vaccinia in non-human primates leads to a $108 \mathrm{~cm}^{2}$ zone of necrosis in 8 days - directly related to cellular destruction from viral replication. We have mutated the virus through insertional deletion of both the thymidine kinase (TK) gene and vaccinia growth factor (VGF) gene. The mutant virus no longer causes destruction of normal tissue, but has completely preserved replication efficiency in tumor tissue and can safely be delivered systematically to successfully treat subcutaneous tumors in mice. Plans are now underway for clinical trials.

Cancer Gene Therapy (2002) 9, 1001-1012 doi:10.1038/sj.cgt.7700549
\end{abstract}

Keywords: vaccinia; replicating; vector; cancer; gene therapy

$\mathrm{M}$ ost modern anticancer therapies are based on the concept that the neoplastic process leads to changes in cellular function that make the tumor cell more sensitive to toxic insults than normal tissue. Modern chemotherapy and radiotherapy exploit a fairly modest window of differential sensitivity, making their therapeutic index small. This small therapeutic index prohibits dose escalation in resistant tumors and limits their effectiveness. The goal in developing new therapies for the treatment of cancer is to develop agents with high toxicity to the tumor cell with minimal pathogenicity to normal tissue. The concept that a virus might contain the necessary discriminatory power to treat human cancers has been around for sometime; however, their exploitation for this purpose has not been feasible until recent advances in our understanding of modern molecular genetics, immunology, and virology. Currently, a variety of custom-tailored viral vectors are under investigation as anticancer agents including adenovirus, herpes simplex virus, reovirus, Newcastle disease virus, and vaccinia virus. Several of these vectors have demonstrated proof of principle in preclinical models and are being tested in clinical trials. Phase I trials have confirmed the safety of these approaches as well as demonstrated some evidence of efficacy.

Received September 24, 2002

Address correspondence and reprint requests to: Dr David L Bartlett, Division of Surgical Oncology, University of Pittsburgh, University of Pittsburgh Cancer Institute, PB02 Cancer Pavilion, 5150 Center Avenue, Pittsburgh,PA 15232,USA.E-mail: bartlettd@msx.upmc.edu
We have chosen to focus on the development of vaccinia virus for the treatment of cancer because we believe that it has advantages as an oncolytic virus over other vectors further along in development. (a) It has a quick, efficient lifecycle, forming mature virions in just 6 hours after infection. (b) It spreads efficiently cell-to-cell, thus increasing the efficacy of in vivo infection. (c) It has a large genome that can accept over $25 \mathrm{~kb}$ of inserted DNA without deletions. In addition, vaccinia virus carries its own strong promoters capable of achieving very high levels of transgene expression. (d) It can infect a wide range of human tissues but does not cause any known human disease. (e) Lastly, there is a large body of knowledge about its biology and extensive experience with it clinically as part of the smallpox vaccination program. Much has been published about the ability of recombinant vaccinia viral constructs to induce an immune response against tumor-associated antigens for the immunotherapy of cancer; however, very few reports have focused on this vector as an oncolytic cancer therapy vector. This report will focus on our experience with the preclinical development of an oncolytic, replication-selective vaccinia virus as an agent for the treatment of human cancers. Specifically, we will discuss the unique features of vaccinia viral biology that make it particularly attractive as an anticancer treatment. We will review our work in creating a tumor-selective variant of the western reserve (WR) strain of vaccinia virus. Lastly, we will examine what we consider to be the critical hurdles to clinical application of this vector, and strategies to overcome these impediments. 


\section{Vaccinia virus biology}

The biology of poxviruses makes them suitable for adaptation to oncolytic vectors. Vaccinia virus is a member of the poxviridae family, which is divided into two major subfamilies, Chordopox (vertebrate pox) and Entomopox (insect pox viruses), based on the host range, sequence homology, and antigenicity. ${ }^{1}$ Vaccinia virus is the prototypical orthopoxvirus that has the longest and most extensive history of use in humans. While Jenner in the 1850s first used the cowpox virus isolated from milkmaids as a vaccine for smallpox, it became clear in the 1930s that the strain being used for the vaccine was genetically distinct from cowpox. Later, this strain was identified as vaccinia. ${ }^{2}$ The precise origin of vaccinia virus is difficult to trace, as it has no known natural host — leading some to suggest that it may have arisen from mutations in the cowpox or smallpox viruses. ${ }^{3}$ However, it is more likely that vaccinia represents a distinct strain that is extinct in its natural host or so rare that it is difficult to identify naturally.

The extensive use of vaccinia virus for both clinical and research purposes has led to the emergence of multiple strains with unique properties. Several of these strains have been fully sequenced and found to contain double-stranded DNA with inverted terminal repeats and a terminal hairpin loop that mimics a large circular single-stranded DNA. ${ }^{4,5}$ The genome consists of 191,636 bp and encodes for 2063 proteins of 65 or more amino acids. These proteins are used to construct the mature virion, which includes a double membrane. The infectious mature virion or extracellular enveloped virus (EEV) is released from the cell by membrane fusion and is responsible for cell-to-cell spread of the virus. The intracellular mature virus (IMV) is released from the cell upon lysis, and is the form of the virus that is produced under laboratory conditions. The EEV is too fragile to withstand the purification process.

The mechanism of vaccinia attachment and uptake into cells is still under intense investigation. This process is likely to involve the $\mathrm{A} 27 \mathrm{~L}$ and $\mathrm{D} 8 \mathrm{~L}$ proteins, which are found in the IMV membrane and appear to bind heparin sulfate and chondroitin sulfate on the cell surface. ${ }^{6,7}$ Fusion is quickly followed by release of viral-transported transcriptional enzymes that transcribe early viral mRNA. These early mRNA typically encode for proteins that are involved with uncoating of the viral DNA and transcriptional factors for intermediate mRNA production. Intermediate mRNA encodes for the late transactivators that lead to late mRNA synthesis. The late proteins include viral structural proteins and early transcriptional factors to be incorporated into the mature virion. ${ }^{8}$ Viral DNA replication occurs, forming concatemers, which are then resolved into individual genomes and assembled into mature virions. The mature virus contains three membranes after assembly; the outer fuses with the cell membrane, resulting in release of a double-membrane viral particle (EEV). The EEV remains attached to the cell surface through the $A 34 R$ gene product, allowing for cell-to-cell spread of the virus without release into the bloodstream. ${ }^{9}$ The EEV form is resistant to antibody neutralization.

It is interesting to note that the vaccinia virus, like the other poxviruses, spends its entire lifecycle in the cytoplasm and has never been shown to integrate into the genome. ${ }^{8}$ Vaccinia has very few interactions with host cellular proteins, allowing for rapid, efficient replication without negative effects from host cell defenses. The virus induces a profound cytopathic effect very soon after viral entry, as early viral enzymes completely shut down host cell function. By 4-6 hours after infection, there is almost complete inhibition of host protein synthesis. This allows for very efficient expression of viral genes and viral replication. In fact, approximately 10,000 copies of the viral genome are made within 12 hours of infection; half of these are incorporated into mature virions and released.

The success of vaccinia as an oncolytic vector relies on its efficiency in vivo. This is dependent on the rapidity of replication and spread, and evasion of host defenses. Vaccinia has developed a wide range of immune evasion strategies in order to survive in vivo. Understanding and manipulating these factors may optimize the vector for clinical use. The genome of vaccinia virus and several other pox members encode for multiple factors that are able to subvert the host immune response (Table 1). If one examines these factors closely, it is clear that the majority of them encode for proteins that are able to actively suppress both innate immunity and the development $\mathrm{T}$ helper 1 (Th1) immune response. For example, vaccinia virus has adopted at least three different genes that can block the function of the interferon (IFN) family members IFN $-\alpha$ and IFN- $\beta .^{10-12}$ These factors are secreted by a variety of cells in response to innate danger signals. They can induce an antiviral state and

Table 1 Production of immunosuppressive molecules by vaccinia virus

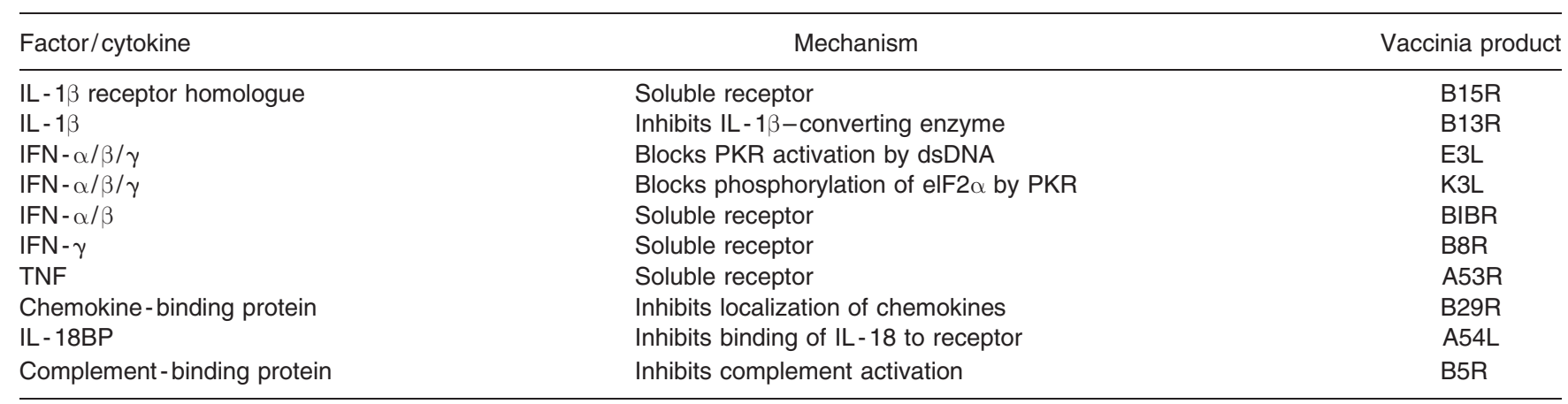


up-regulate adaptive immune functions. Vaccinia also carries genes for multiple inhibitors of chemokines - some of the earliest substances produced during the initiation of an immune response. ${ }^{10-12}$

In addition to actively suppressing early innate responses to viral infections, vaccinia also produces several factors that are able to inhibit the development of a Th1 immune response. Vaccinia encodes for at least three factors that can directly block the function of IFN $-\gamma$, one of the most potent Th1 cytokines. ${ }^{13,14}$ In addition, vaccinia also encodes for the recently described IL-18-binding protein (IL-18BP). ${ }^{15,16}$ $\mathrm{IL}-18 \mathrm{BP}$ is a naturally produced soluble factor that blocks the binding of IL-18 to its cognate receptor. IL-18BP has been shown to be one of the most potent inhibitors to the development of a Th1-biased immune response. ${ }^{17}$ In addition, vaccinia virus also encodes for several other immunosuppressive factors including factors to block complement activation, IL - $1 \beta$ soluble receptor, and soluble TNF receptor antagonist. $^{18-21}$ These observations suggest that subverting the early innate immune response and slowing the development of Th1 responses are important for the efficacy of oncolytic therapy. This has been confirmed in several different models of vaccinia infection. For example, the modified vaccinia virus Ankara (MVA) strain is an attenuated strain of vaccinia that was created by serial passage through chick embryo fibroblasts. MVA replicates poorly in mammalian cells demonstrating markedly diminished virulence. $^{22}$ The MVA genome has been sequenced and found to contain six large genomic deletions that include loss of the genes that encode for inhibition of IFN $-\alpha$, IFN- $\beta$, FIN $-\gamma$, TNF, and chemokines, which are felt to be responsible for its striking decrease in virulence. ${ }^{23}$ Similarly, Deonarain et al ${ }^{24}$ have shown that IFN $-\alpha$ and IFN- $\beta$ knockout mice demonstrate markedly enhanced susceptibility to vaccinia viral infection.

Other studies have confirmed the critical role of Th1 response to clearance of vaccinia viral infection. Van Den et $\mathrm{al}^{25}$ examined the effect of Th1 (IFN- $\gamma$, IL-12) and Th2 (IL-4, IL-10) balance in the clearance of vaccinia virus in mice using cytokine knockouts. Vaccinia viral replication was enhanced in IL - 12 and IFN- $\gamma$ knockout mice, with IL$12(-/-)$ demonstrating greater susceptibility to infection than IFN- $\gamma$-deficient mice. Interestingly, development of antivaccinia CTL was completely abrogated in IL-12 knockout mice but remained normal in $\operatorname{IFN}-\gamma(-/-)$. In contrast, IL-4- and IL-10-deficient mice showed marked enhancement of vaccinia viral clearance, suggesting that these cytokines naturally suppress the host response to vaccinia. IL $-10(-/-)$ mice exhibited greater inhibition of viral replication than IL-4-deficient mice. Interestingly, when the effects of each of these cytokines on vaccinia infection were examined in recombinant viral constructs, local expression of IL - 4 showed a much greater inhibition of host responses. In fact, while the absence of IL-10 resulted in improved clearance of vaccinia virus that was mediated by increased levels of IL- 6 and IL-1, the local expression of IL - 10 had little to no effect on viral clearance.

In summary, these studies confirm the importance of the development of Th1 response to successful clearance of vaccinia virus. Taken together, all of these data confirm the important role of both the innate and the Th1 response play in the clearance of vaccinia virus. These findings also are critically important to the continued development of vaccinia viral vectors for the treatment of cancer for, as we will review later, one of the chief obstacles to the clinical application of these vectors for the treatment of human cancers is premature clearance of the virus by the host.

\section{WR strain vaccinia virus as a tumor-selective oncolytic virus}

Perhaps the most critical component of a successful oncolytic virus is the ability to induce tissue destruction. The characteristic lesion created by the smallpox vaccine (vaccinia) in immunosuppressed hosts is known as vaccinia necrosom, consisting of a spreading necrotic ulcer as a direct result of replicative necrosis. We focused on a strain of vaccinia (WR) that is considered more efficient than the vaccine used clinically. We observed that $10^{6}$ pfu of a wildtype WR strain of vaccinia, injected intradermally in rhesus macaques, led to a necrotic ulcer of $108 \mathrm{~cm}^{2}$ in diameter in only 8 days, without systemic spread of the virus (unpublished data). This ability to quickly spread, express genes, and effectively destroy tissue is unique among current oncolytic viral vectors in clinical and preclinical development. We hypothesize that a tumor-selective replicating form of this strain of vaccinia would have real potential as an "oncolytic" virus. We further hypothesize that combination of a tumor-selective variant of this strain with either immunostimulatory genes or suicide genes that could enhance this oncolytic effect would form the basis for an effective treatment for human cancers.

\section{Development of tumor-selective mutant vaccinia}

Our initial efforts have focused on mutating the WR strain of vaccinia virus to make it replication-selective in tumor cells. We and others have studied the wild-type virus and found that after intravenous injection, the highest amount of virus can be recovered from the tumor, the second highest from the ovary, and minimal to no virus is recovered from other organs. $^{26,27}$ The natural tropism of this virus to tumor is surprising and the mechanism of this is not established. Historically, smallpox virus was noted to have tropism for injured and irritated skin. ${ }^{28}$ This is thought to be secondary to histamine release, leading to leaky vasculature allowing for transfer of the virus out of the circulation. Vaccinia is a large virus particle ( $350 \mathrm{~nm}$ in diameter) and would require leaky vasculature for extravasation into tissues. Notably, the tumor and ovarian follicles are both known to be sites of vascular endothelial growth factor (VEGF) production and leaky vasculature. ${ }^{29}$ We have demonstrated by immunohistochemistry that vaccinia tropism to the ovary is specific for ovarian follicles. ${ }^{30}$

We first studied a virus with the thymidine kinase $(T K)$ gene deleted. ${ }^{31}$ This deletion forces the virus to be dependent on host cell nucleotides, which are more available in dividing cells compared to resting cells. This TK virus demonstrates tumor selectivity over other tissues. This was demonstrated 
in numerous tumor models, including murine colon cancer and melanoma, rat sarcoma, human colon cancer in nude mice, and rabbit kidney cancer. ${ }^{32}$ As demonstration of the selectivity and efficiency, we showed that this virus can be injected intravenously or intraperitoneally and be taken up into a subcutaneous tumor, replicate in the tumor, and lead to an antitumor response. ${ }^{33}$ This effect is directly related to intratumoral vaccinia replication, and not a bystander inflammatory response. The effect is enhanced in athymic/ nude mice, which cannot mount an effective immune response against the virus ${ }^{34}$ (Table 2 ). As we will discuss later, the effect can be enhanced with enzyme/prodrug suicide gene systems ${ }^{35}$ and by using hyperthermia to increase vascular permeability and increase viral extravasation into the tumor (unpublished data).

In order to further improve the specificity of this virus for tumor replication, we mutated the vaccinia growth factor $(V G F)$ gene in parallel with the $T K$ gene. ${ }^{36} \mathrm{VGF}$ is a protein that is expressed early by vaccinia virus and is secreted by infected cells. It binds growth factor receptors on surrounding resting cells and stimulates them to proliferate. ${ }^{37}$ This will increase the available nucleotides in these resting cells, thus compensating for the loss of viral TK. We hypothesized that deleting both the $T K$ and $V G F$ genes would lead to near complete abrogation of replication in resting cells and further attenuate the virus, without decreasing the ability of the virus to replicate in the tumor environment. The double-deleted virus was made and tested, and found to have markedly enhanced tumor specificity (Figs 1 and 2 ). This virus could be given systemically at doses of $10^{8}$ pfu to a nude mouse without pathogenicity, and led to significant regression in established subcutaneous tumors (Fig 3). ${ }^{36}$ This doubledeleted virus was tested in rhesus macaques and was found to be completely nonpathogenic when delivered intravenously at doses up to $10^{9} \mathrm{pfu}$ (unpublished data). Intradermal inoculation at $10^{6} \mathrm{pfu}$ demonstrated no viral replication (compared to the $108 \mathrm{~cm}^{2}$ necrotic ulcer from the wild type). Nevertheless, 4 days after intravenous virus delivery, equal titers of wild-type and double-deleted virus could be recovered from subcutaneous tumors in mice. The doubledeleted virus demonstrated marked attenuation in vivo with preserved intratumoral replication. This mutated virus shows significant promise and two of these vectors have been made in clinical grade for clinical trials - one with a suicide gene and somatostatin receptor imaging gene for clinical trials.

One limitation to mutating a virus for selective replication in dividing cells is potential toxicity to other dividing cells in vivo. Vaccinia can efficiently infect almost all cell types in vitro, and it is hard to imagine delivering this systemically to animals or humans without significant pathogenicity. Other sites of dividing cells known to suffer toxicity from chemotherapy agents such as bone marrow-derived cells and gastrointestinal mucosa are not affected by systemic vaccinia in our murine, rat, rabbit, or primate studies. We hypothesize that the large virus requires a leaky vasculature for extravasation into tissues in addition to proliferating cells. This leaky vasculature is lacking in the GI mucosa. Despite bone marrow-derived cells having ready access to circulating vaccinia, no bone marrow toxicity is encountered, even in animals succumbing to viral pathogenicity. ${ }^{36}$ Bone marrow-derived cells are not infected well by vaccinia for unknown reasons. The one place that we do recover replicating virus is from ovarian follicles. The developing follicle is much like a tumor with developing, leaky vasculature, high levels of VEGF, and replicating cells. ${ }^{29}$ Immunohistochemistry demonstrated that the double-deleted vaccinia replicated efficiently throughout developing ovarian follicles, without infection or spread through normal ovarian parenchyma. The wild-type virus, however, spread throughout the ovarian parenchyma and destroyed the ovary. Primates have much fewer developing follicles, and we could not recover any virus from the ovaries of rhesus macaques after intravenous injection.

We have demonstrated both safety and efficiency with a double-deleted WR vaccinia virus in murine models and safety in the rhesus macaque model. However, the challenge of large, slow-growing human tumors may be greater. A virus mutated for selective replication in dividing tumor cells may not replicate as efficiently in slow-growing human tumors compared to the murine tumor models (although our experience with slow-growing human tumor xenografts in nude mice suggest that the vector will be effective). Therefore, it would be helpful to have other strategies to enhance the tumor selectivity of the vector.

\section{Creation of viral mutants that take advantage of transformed phenotype}

Virus mutations, which take advantage of the transformed phenotype and innate cellular resistance to apoptosis rather than the rate of cell division, may demonstrate enhanced efficiency in slow-growing tumors. The natural host cellular response to viral infection is apoptosis; therefore, viruses have evolved with genes, which encode for proteins that inhibit cellular apoptosis. The E1B protein of adenovirus plays such a role by binding to p53 and inhibiting its function. E1B-deleted adenovirus has diminished ability to replicate in normal host cells, but preserved replication in

Table 2 Tissue luciferase activity (RLU/mg protein) after intravenous delivery of $10^{6}$ vaccinia luciferase

\begin{tabular}{|c|c|c|c|c|}
\hline Tumor model & Tumor & Ovary & Liver & Lung \\
\hline $\begin{array}{l}\text { Adenocarcinoma liver metastases } \\
\text { in immunocompetent mice }\end{array}$ & $46,000,000$ & 1450 & - & 600 \\
\hline Subcutaneous sarcoma in rat & 4,337 & 0.74 & 0.023 & 0.056 \\
\hline VX-2 liver metastases in rabbit & 2,103 & 132 & 9 & 7 \\
\hline Human melanoma in athymic mice & 558,000 & 8000 & 215 & 963 \\
\hline
\end{tabular}




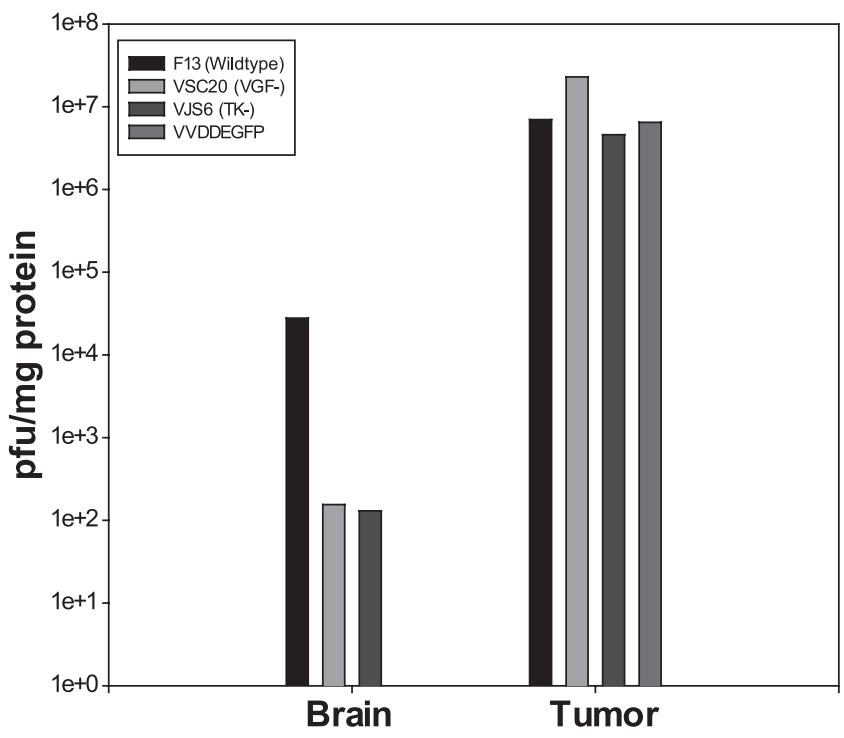

Figure 1 Vaccinia titers recovered from brain and tumor 4 days after injection of virus intraperitoneally in MC38 subcutaneous tumorbearing mice. Data represents the median of 5 values. Of note, no recoverable titers are seen in brain tissue from the double deleted virus (VVDDEGFP), while the tumor has equivalent titers to wild type.

tumor cells. ${ }^{29,38}$ While there has been noted controversy with the exact mechanism, the concept is sound and may be studied in other viruses. Poxviruses have some well-known inhibitors of apoptosis. The most widely studied is the $\mathrm{CrmA}$ gene from cowpox, which inhibits ICE, a downstream mediator of apoptosis. ${ }^{29,39}$ The vaccinia homologue of this gene encodes for the serpin, SPI-2. ${ }^{40}$ This and a similar protein, SPI-1, have both been shown to inhibit cellular apoptosis and have been deemed "host range genes" based on limited in vitro replication in defined host cell lines after their deletion. ${ }^{29}$ There has been much studied and continued controversy over the role of apoptosis in vaccinia replication. Much of what has been reported has been in transformed cell lines in vitro, which likely have inherent resistance to apoptosis. $^{41}$ In vivo studies are lacking. Also conflicting is the fact that viral-induced apoptosis is usually through a different pathway (e.g., IFN signal transduction) than that taken advantage of by suppressor gene mutations in tumors (e.g., p53). Nevertheless, we hypothesized that these host range genes may play a role in viral replication in nontransformed cells, and their deletion would therefore provide a selective advantage to tumor cells. Our preliminary studies have supported this hypothesis. This advantage would be best borne out in in vivo studies. In addition to SPI1 and SPI-2, four other host range genes have been identified. ${ }^{42}$ We are currently studying $K 1 L$ and $C 7 L$ deletion mutants alone and in combination with Spi deletions in vitro and in vivo. ${ }^{43-45}$

\section{Creation of tissue-specific vaccinia viral recombinants}

One obvious means of increasing tumor specificity and decreasing pathogenicity of vaccinia is to alter the viral surface proteins. The mechanism of uptake of vaccinia virus into cells is not clearly understood. There is no defined receptor for vaccinia virus uptake. The purified form of virus used in clinical trials is the intracellular mature virion (IMV), whereas cell-to-cell spread of the virus in vivo occurs through the extracellular enveloped form of the virus (EEV). Other viruses have been mutated to alter viral tropism or improve infection of resistant cells. Vaccinia has natural tumor tropism and infects all tumor cells well. Our goal was to alter the EEV coat such that spreading infection in vivo can only occur in tumor tissue and not in normal tissue. This would greatly enhance the safety of the vector.

Numerous proteins have been identified on the surface of the EEV. One in particular, the A34R, appears to play a significant role in viral release from cells as well as viral infection of neighboring cells. ${ }^{9}$ A34R is a type II transmembrane protein with the $\mathrm{C}$-terminus in the extracellular domain. Virus that is deleted of this gene fails to form normal-sized plaques in vitro and is markedly attenuated in vivo. We generated vaccinia shuttle plasmids creating fusion genes with a full-length $A 34 R$ gene or a truncated (148 nucleotides) form of the gene fused to a $\mathrm{scFv}$ specific for ovarian cells (MOvy specific for folate-binding protein, FBP). The truncated form lacked the lectin binding domain, which was demonstrated to be essential for normal cell-tocell spread of the virus. We recombined these fusion genes into vaccinia such that the native $A 34 R$ gene was replaced with the fusion genes. We compared the wild-type, $A 34 R$ deletion mutant, A34R-Mov full-length (M168), and A34R-Mov truncated (M148) for infectivity, plaque morphology, growth curves, and immunofluorescence in different cell lines with or without the FBP. We found that the truncated fusion protein was attenuated in cells lacking the FBP, with a similar phenotype to the A34R-deleted virus. The full-length $A 34 R$ fusion mutant had a similar phenotype to the wild-type virus. No advantage could be demonstrated for replication in FBP-positive cell lines. While the Mov antibody fusion constructs were stable, we were unable to demonstrate specific binding to FBP-expressing cells, FBP bound to plates, or a MOv anti-idiotype antibody. The fusion was, therefore, nonfunctional (unpublished data). Other investigators have established stable production of EEV coat protein fusion antibodies without a change in viral tropism. ${ }^{46}$ Future investigation of this strategy awaits a better understanding of the precise molecular mechanisms that regulate uptake of vaccinia virus.

\section{Targeting vaccinia to solid tumors with local hyperthermia}

We hypothesized that hyperthermia may augment vaccinia delivery to tumors after systemic injection, as hyperthermia increases the permeability of the endothelial vasculature to nanoparticles. We first demonstrated that hyperthermia does not alter tumor cells' susceptibility to the intrinsic cytopathogenicity of the vaccinia virus compared to normothermic controls. Hyperthermia also does not change the viral infectivity or the level of viral marker gene expression when compared to normothermia. We then utilized an in vitro model of endothelial cell monolayer permeability, and 
Brain
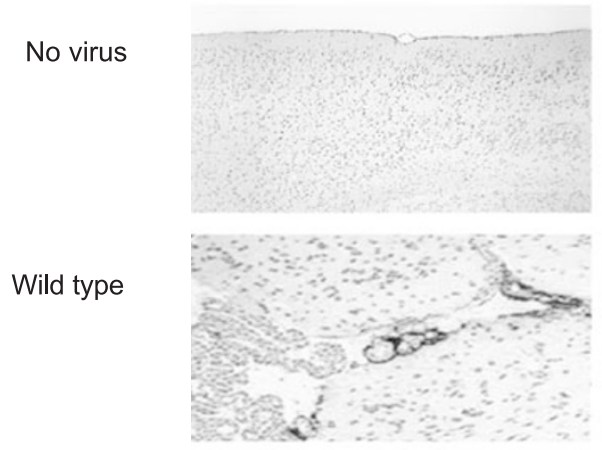

TK-

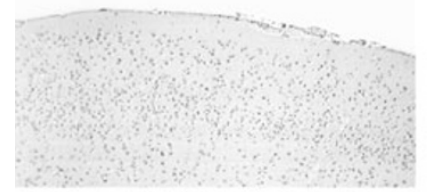

VGF-

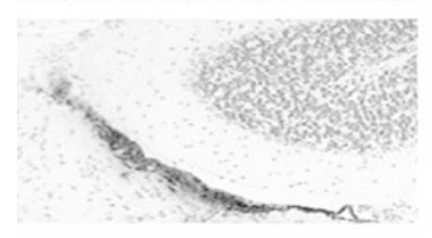

TK/VGF-
Ovary
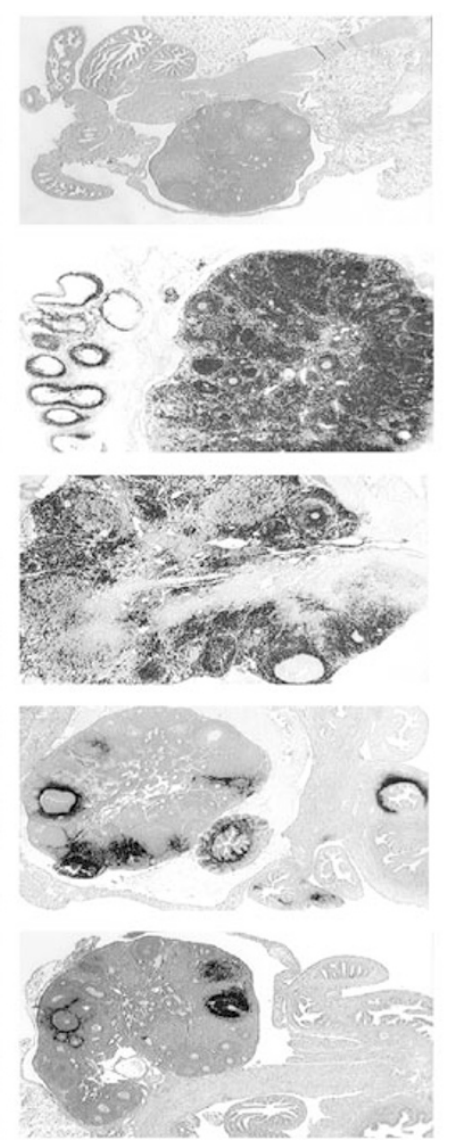

Tumor
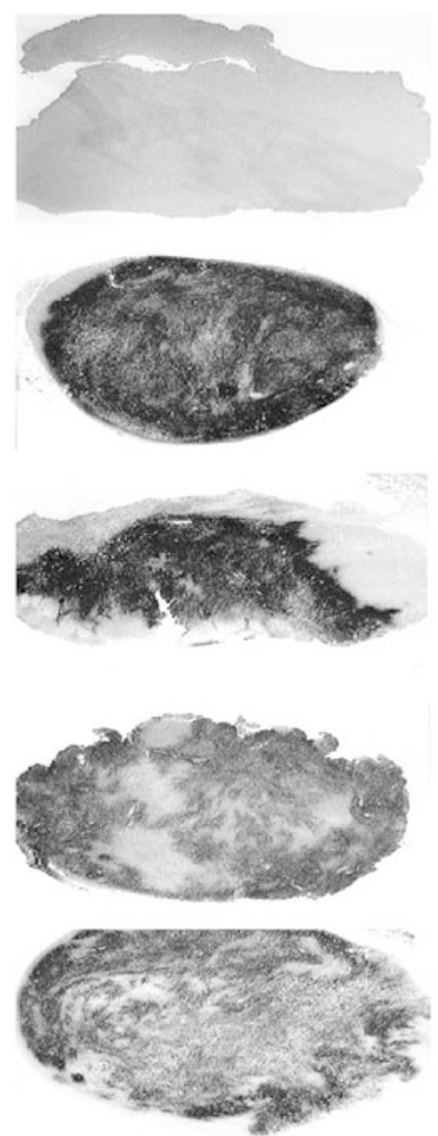

Figure 2 Immunohistochemistry using an anti-vaccinia antibody in tumor, ovary, and brain after intraperitoneal virus delivery in a murine subcutaneous tumor model. Diffuse staining of the tumor is demonstrated with all viruses. The mutant vaccinias demonstrate selective infection of ovarian follicles, compared to the diffuse staining with non-selective viruses. No staining in the brain could be demonstrated with the double deleted virus. ( Courtesy of Dr. Andrea McCart)

demonstrated that hyperthermia increases the permeability of the monolayer to vaccinia virus and that this phenomenon is completely reversible. Next, we tested viral delivery, marker gene activity, and antitumor response in vivo with regional delivery of hyperthermia to a subcutaneous flank tumor (MC-38) in immunocompetent mice. We demonstrated that the tumors, which were treated with systemic vaccinia under conditions of hyperthermia $\left(41.5^{\circ} \mathrm{C}\right.$ for 30 minutes), had significantly higher levels of vaccinia marker gene activity ( $>100$-fold) than those treated under normothermic conditions and that this effect was specific to tumor. We also demonstrated that mice with $1-\mathrm{cm}$ subcutaneous tumors treated with a systemically delivered conditionally replicating vaccinia under conditions of hyperthermia had complete tumor regression in $50 \%$, and significantly improved antitumor response compared to normothermic viral-treated controls (mean tumor volume of 110 vs $3169 \mathrm{~mm}^{3}, 13$ days after treatment), and compared to hyperthermic, nonvirally treated control animals (unpublished data). Regional hyperthermia improves vaccinia targeting to tumors, and thereby enhances the antitumor response. This suggests that viral extravasation is one ratelimiting step for successful treatment, and that tissues with a baseline leaky vasculature (such as tumors and ovarian follicles) may have increased susceptibility to systemically delivered. We have now begun testing regional delivery of vaccinia virus under hyperthermic conditions in primate models. Preliminary observations indicate that this method is safe and effective and can prevent systemic spread of the virus.

In summary, we have developed a highly tumor-specific strain of the WR vaccinia virus. We have shown that this virus in murine models, when given systemically, is able to target tumor cells, infect and express its gene products, and mediate antitumor effects. Furthermore, we have preliminary data suggesting that this double-deleted variant does not demonstrate pathogenicity for normal tissues in primates. The search for additional host range genes, as well as other methods that may allow for more selective replication in tumor cells, will continue. However, it may be that very little additional tumor selectivity is necessary. Therefore, we have turned our attention to other aspects of this system that we perceive to be the impediments to its use clinically. Specifically, two additional hurdles must be dealt with in order to develop an effective antitumor treatment using this vector: (a) regulation of 


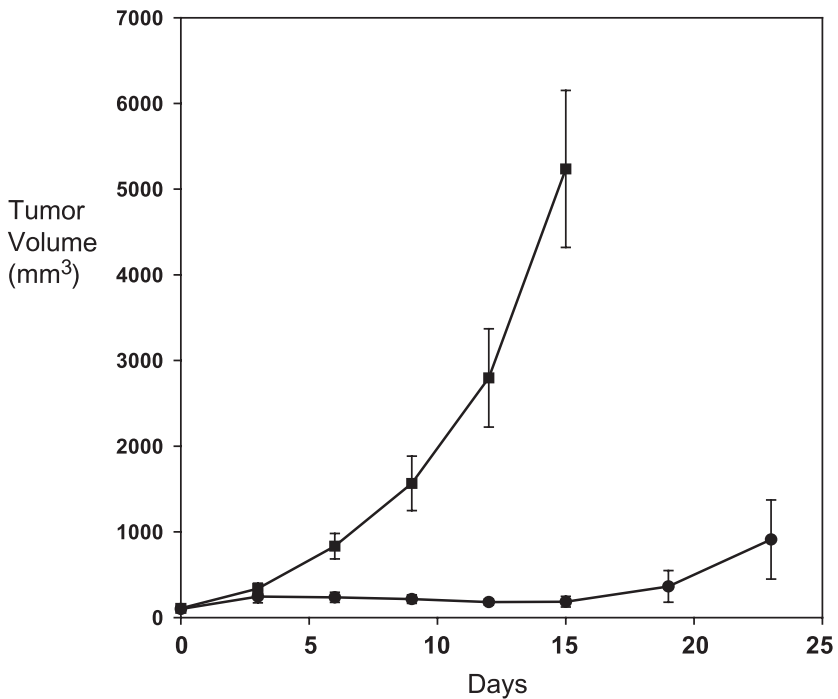

Figure 3 Tumor volume after systemic treatment with $10^{9} \mathrm{pfu}$ of the double deleted vaccinia on day 0 compared to PBS control in established subcutaneous MC38 tumors. Significant inhibition of tumor growth is present as a result of vaccinia replication in the tumor.

immune clearance of the vector, and (b) improved bystander cytotoxicity.

\section{Improving in vivo replication efficacy of vaccinia virus by regulating immune clearance}

The immune response to vaccinia viral vectors serves as our paradoxical friend and foe in attempting to develop them into an effective antitumor therapy for human cancers. However, the vigorous immune response is desirable because we believe that with the appropriate guidance, it can be directed to enhance immune-mediated destruction of local and distant noninfected tumor cells (see below). However, the vigorous immune response leads to premature clearance of the virus before adequate levels of tumor infection have occurred, thus decreasing the level of transgene expression and possibly the overall antitumor efficacy. Our early experience with vaccinia virus has confirmed this to be the case. When we compared infection/replication efficiency in immunocompetent and nude mice after systemic injection of vaccinia-GFP to subcutaneous tumors (Figs 4 and 5), a higher percentage of cells expresses GFP in athymic/nude mice after 48 hours (up to $60 \%$ of cells express GFP). This problem is especially true in the preimmunized host where our experience has indicated that early clearance of the virus by the host immune response severely limits the antitumor efficacy. There are several strategies that have been investigated to circumvent this problem of premature immune clearance.

First, one could create a virus that is less recognizable by the immune system. This could be accomplished by mutating the viral coat of the vaccinia virus to make it less cross reactive with antibodies. However, the poxviridae — and in particular vaccinia virus — is antigenically very complex and it is unlikely that one or two mutations in viral envelope genes could significantly alter antibody recognition. Further, any mutations in the viral envelope may decrease the infectivity of the virus.

Another strategy would be to develop other poxviruses that are able to selectively infect and lyse human tumor cells that do not cross-react with vaccinia. We have initially investigated one such vector, the yaba-like disease (YLD) virus. ${ }^{47}$ The Yabapox genus has species that replicate in human cells and cause limited skin lesions similar to vaccinia inoculations, but do not cross-react with vaccinia virus. The natural host for these viruses is unknown, but they have been isolated from skin lesions in monkey colonies. Almost no prior work had been reported with this virus, so we characterized the virus in terms of plaque morphology, host range, and replication efficiency. We found that we could grow the virus in high titers, similar to vaccinia virus, under normal conditions using CV-1 (monkey kidney) cells. We discovered that the host range for YLD was more restrictive than that for vaccinia. The virus replicated well in monkey and human cell lines but not in rodent cell lines. The infectivity and plaque formation were a slower, less efficient process than that of vaccinia virus, even in permissive cells. We performed ELISA on YLD-coated plates using vaccinia antiserum and found no reactivity, verifying the supposition that no antibody cross-reactivity exists between vaccinia and YLD. We also performed experiments using a plasmid with the vaccinia synthetic promoter expressing $\beta$-galactosidase, which was transfected into YLD-infected cells, demonstrating that the YLD polymerase recognized the vaccinia promoters. In order to recombine a marker gene into the YLD virus, we sequenced the YLD TK gene. Not enough TK homology exists between pox genera to allow for efficient recombination using the vaccinia TK sequence. We created a YLD shuttle plasmid and recombined GFP into the TK locus of YLD. We demonstrated that the YLD-GFP virus could mediate highly efficient gene transfer and expression in monkey and human cells. This was not as efficient as our vaccinia virus constructs, but much more efficient than other vectors that we had investigated, including adenovirus and liposomemediated gene delivery. This YLD virus was tested in vivo. Nude mice with established A2780 human ovarian peritoneal carcinomatosis were infected with YLD virus at $10^{8}$ pfu injected intraperitoneally. Tumor was sampled every 4 days and FACS analysis was performed for GFP expression. By 12 days, 20\% of tumor cells expressed GFP (Fig 6). YLD is a potentially useful vector for vaccination or local injection of tumors, as it appears to be more efficient than other vectors being studied in this regard, but it remains much less efficient than vaccinia virus.

Another approach to circumventing premature clearance of our vaccinia vector is to create a viral recombinant that actively suppresses host cellular immune responses. Several groups have reported that insertion of $\mathrm{Th} 2$-like cytokines such as IL-4 or IL-10 into vaccinia virus increases in vivo viral replication and slows host clearance of infection. ${ }^{25,48}$ However, creation of a virus that is not recognized by the immune system obviously creates serious safety concerns for the population as a whole, as unforeseen events could 


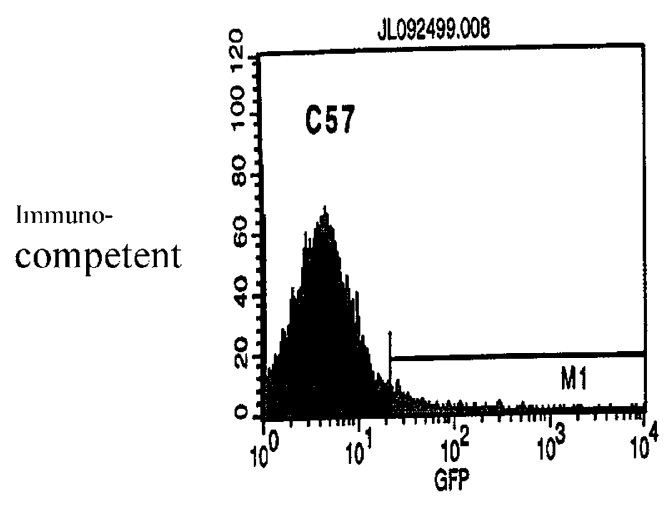

Control

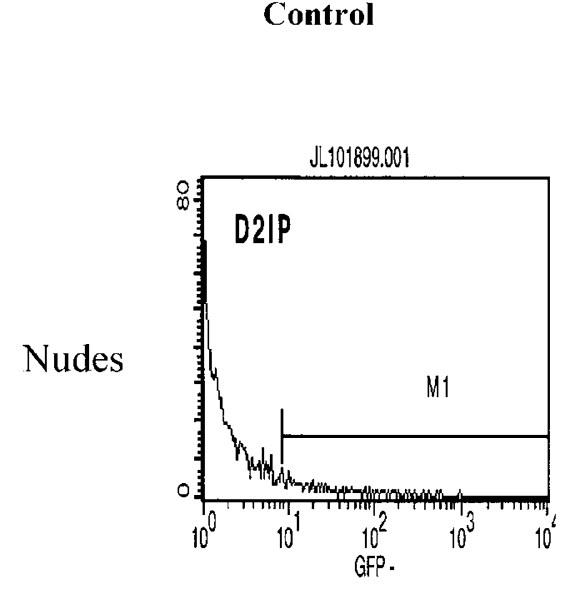

Control

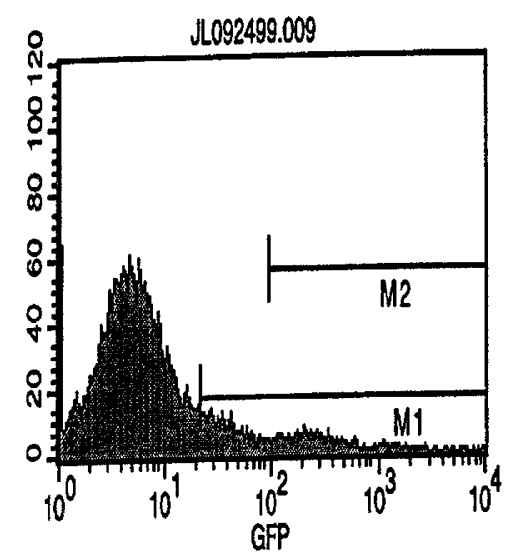

Day 2

$13.18 \%$

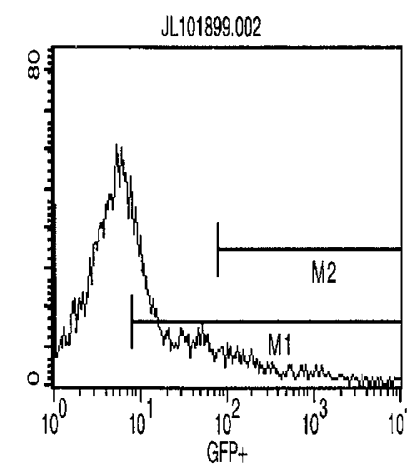

Day 2

$37.9 \%$

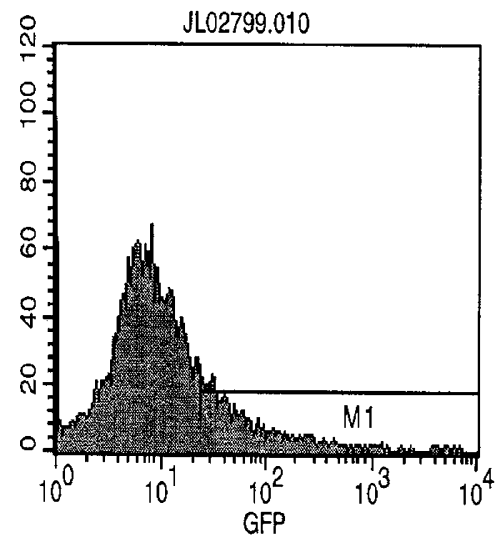

Day 4

$15.72 \%$

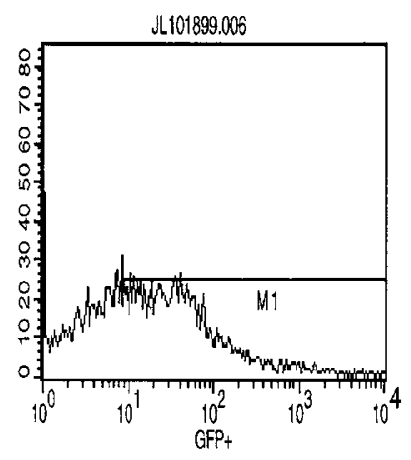

Day 4

Figure 4 Tumor FACS from Athymic Nude and immunocompetent Mice with MC38 SQ Tumors Treated with $10^{8}$ pfu IP Vaccinia-GFP. Tumors harvested and digested in a triple enzyme solution for 2-3 hours. Single cell suspension analyzed for GFP by FACS analysis. Almost $60 \%$ of cells in the tumor expressed the transgene by Day 4 in the nudes, compared to only $16 \%$ in immunocompetent mice.

lead to a pathogenic virus that is not immunologically cleared.

A third approach to improving in vivo viral replication involves reversible transient host immunosuppression. Because of the growth of knowledge in solid organ transplantation, we now have available multiple immunosuppressive agents that can very precisely target specific pathways of the host immune response. This knowledge, combined with our growing understanding of the immune response to vaccinia virus, should allow us to reversibly slow down the immune response to our vector. This will theoretically allow for more efficient in vivo viral replication in the tumor, higher transgene expression, and greater oncolysis. We hypothesize that this will be a feasible approach with the vaccinia mutant because of the remarkable tumor selectivity that it exhibits. As previously outlined the double-deleted vector demonstrates no pathogenic effect in nude mice when injected systemically at does up to $10^{8} \mathrm{pfu}$.

In fact, our preliminary work in this area has suggested that transient immunosuppression can improve the in vivo replication efficiency and antitumor efficacy of the vector. Our early studies have focused on the depletion of immune cells in order to better understand which effector cells are critical for allowing efficient in vivo infection of tumor cells. Antibodies to CD4 and CD8 murine T cells were administered to tumor-bearing mice (subcutaneous MC-38), individually or combined, to deplete both populations of $\mathrm{T}$ cells. T-cell depletion was verified by FACS analysis. TKdeleted vaccinia expressing $\beta$-galactosidase was then delivered intraperitoneally and $\beta$-galactosidase expression in the subcutaneous tumor was analyzed. T cell-depleted animals demonstrated a higher peak gene expression level compared to nondepleted animals. Intact animals eliminated the virus (and $\beta$-galactosidase expression) within 10 days, whereas depleted mice demonstrated prolonged expression throughout the duration of the study. CD4- and CD8 expressing $\mathrm{T}$ cells contributed equally to immune clearance of vaccinia, and the most prolonged expression was achieved with the combined depletion. We measured $\operatorname{IgG}$ and $\operatorname{IgM}$ formation against vaccinia and, as expected, we demonstrated no isotype switch to IgG in the T cell-depleted host. This finding may eventually be very important as it may allow for repeat administration of the virus. Finally, we examined tumor responses after delivery of $10^{9}$ pfu of 


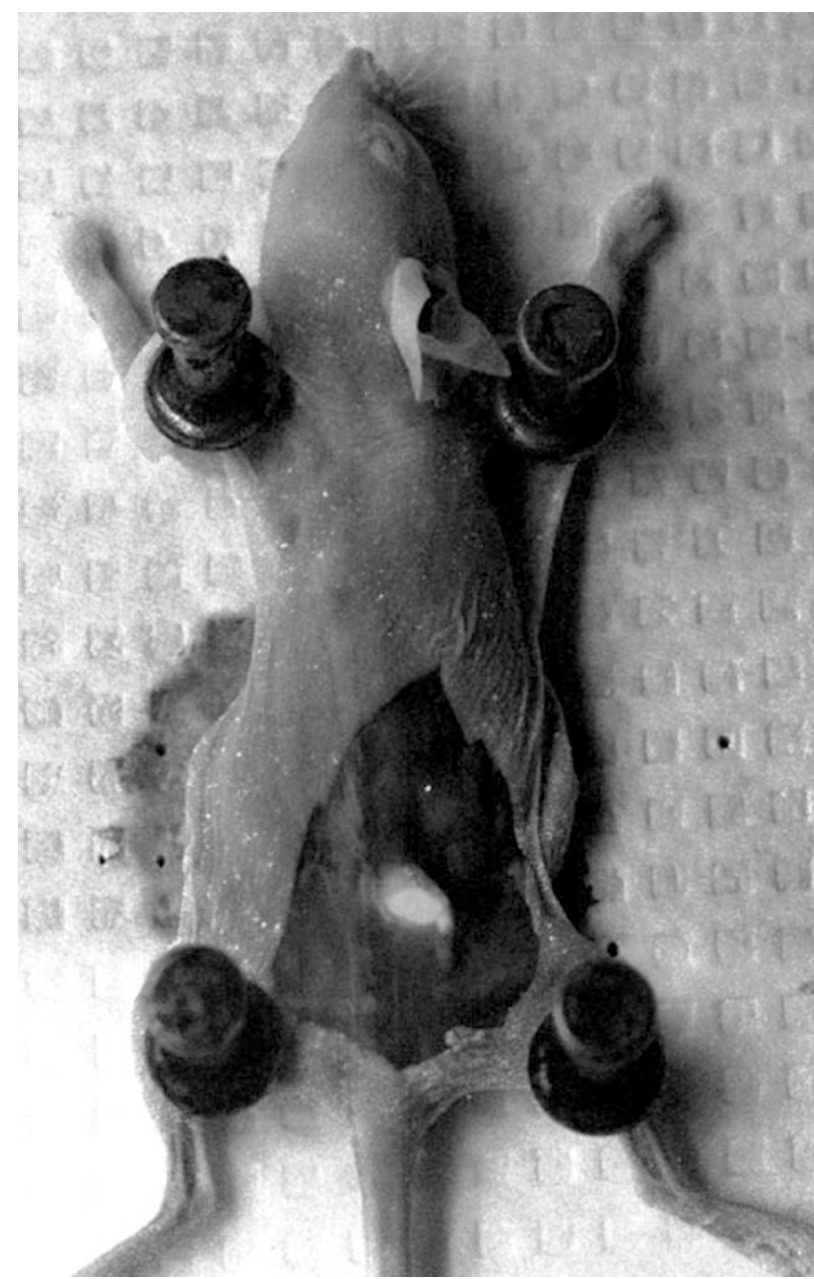

Figure 5 GFP flourescence is specific for this subcutaneous tumor after intravenous injection of vaccinia-GFP.

vaccinia intraperitoneally. We demonstrated significantly improved inhibition of tumor growth in $\mathrm{T}$ cell-depleted animals, compared to intact animals.

Our experience has also suggested that successful reinfection of a preimmunized host appears to be possible through transient immunosuppression. In a pilot study, when a rhesus macaque was preimmunized with wild-type WR vaccinia virus and reinfected with the same virus, no live virus could be recovered form the animal. However, if these same animals (with high circulating $\operatorname{IgG}$ antibodies against vaccinia) were pretreated with FK-506, prednisone, cyclophosphamide, and complement inhibitor and then reinfected with wild-type virus, the virus spread quickly and was recoverable from the blood and other organs (unpublished data).

\section{Improving antitumor effects of vaccinia viral-mediated therapy through "bystander effects"}

Because $100 \%$ infection and "oncolysis" of all tumor cells, even with transient immunosuppression, is unlikely to be achieved in vivo, a successful viral-mediated treatment of cancer must employ some mechanism for bystander cytotoxicity. There are several strategies to creating a bystander effect that are shared by most viral vectors currently being investigated for the treatment of cancer: (a) incorporation of a toxic transgene into the vector that will allow for killing of uninfected surrounding tumor cells, and (b) the use of immunostimulatory genes to create an in vivo oncolysate vaccine.

We have investigated both the purine nucleoside phosporylase gene and the cytosine deaminase gene in our vaccinia system. We have demonstrated significant antitumor effects with each of these systems in a murine model of hepatic metastases $^{33,49}$ (Gnant et al, unpublished data). We have also observed that the interaction between the oncolytic effect of the replicating virus and the enzyme/prodrug is quite complex. However, the virus itself can directly infect and kill tumor cells. Nevertheless, the cytotoxic effect of the enzyme/prodrug can directly decrease viral replication. We have explored this relationship in the vaccinia-CD system to better define the advantageous and detrimental interactions between the replicating oncolytic effect of vaccinia virus and the enzyme/prodrug therapeutic effect. ${ }^{33}$ In vitro infection of cancer cells with vaccinia-CD at high $\mathrm{MOI}(>0.1)$ led to cell death secondary to a viral cytopathic effect. Addition of the prodrug had no added effect. At low MOI, no viral alone effect was observed, but the prodrug mediated significant cell death. Importantly, cell lysates demonstrated 300 -fold reduced viral recovery from cells treated with both vaccinia$\mathrm{CD}$ and 5-FC, compared to vaccinia treatment alone. It

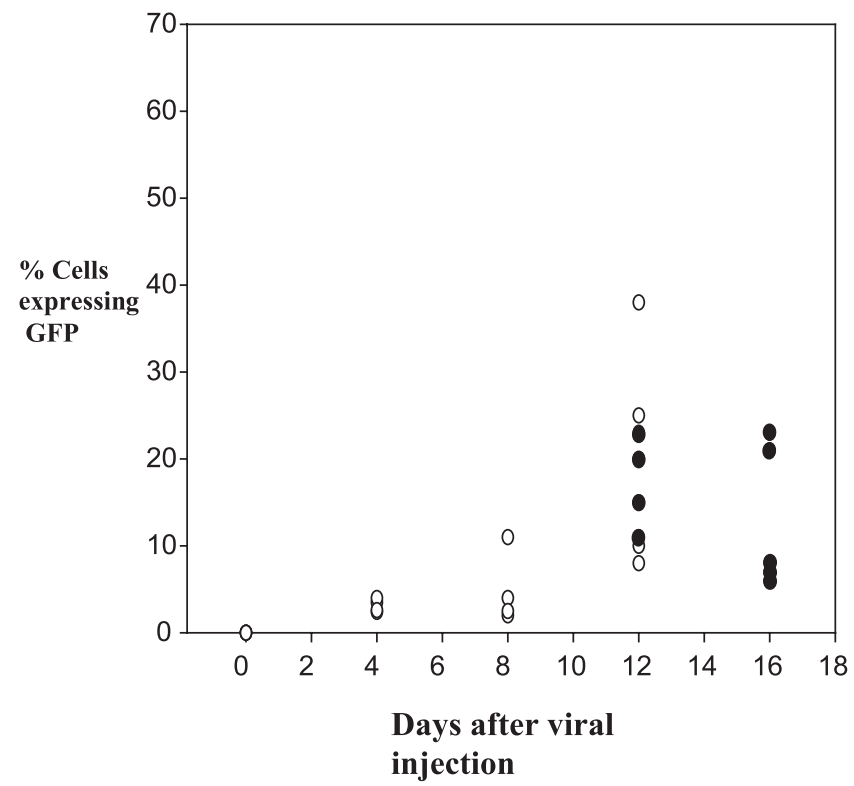

Figure 6 In vivo tumor GFP expression after infection with YLD virus. The graph represents the percent of cells expressing GFP on the various days of sacrifice. Two experiments were performed, one with a 4 week tumor burden (5-7mm tumor nodules). These results are shown in the open circles. The second experiment was performed with two week old tumors ( 1 to $2 \mathrm{~mm}$ nodules). These data points are shown in the filled circles. Each circle represents a single animal. There are at least 4 animals per group. On some days the circles overlap. 
appears that the converted prodrug (5-FU) has inhibitory effects on viral replication, either by direct interference with viral DNA synthesis or by killing surrounding cells prior to infection by vaccinia virus. We explored this interaction further in vivo in a model of subcutaneous tumor in the nude mouse. Mice injected with vaccinia-CD (with or without 5-FC treatment) had smaller tumors than the controls, suggesting that replicating vaccinia alone is cytotoxic to tumors in vivo, even after systemic injection $\left(10^{8} \mathrm{pfu}\right)$. The addition of 5-FC improved the antitumor response when a low dose of virus was injected into tumors $\left(10^{7} \mathrm{pfu}\right)$, leading to $50 \%$ complete regressions of subcutaneous tumors. $5-\mathrm{FC}$ treatment resulted in no antitumor advantage after intraperitoneal delivery of $10^{8} \mathrm{pfu}$, compared to virus alone. Of note, compared to mice that received virus alone, those that received vaccinia-CD plus $5-\mathrm{FC}$ had significantly prolonged survival from viral-mediated death. The addition of the enzyme/prodrug system to a replicating vaccinia virus can improve the antitumor response and decrease viral pathogenicity; however, the complex nature of theses highly interactive systems will require further study. One potential area for improvement would be the development of a vaccinia-specific inducible expression system that would allow for the tight regulation of the toxic transgene.

Another strategy for increasing the bystander effect is through expression of factors that drive an immunologic response to the uninfected tumor cell. This strategy would maintain the high discriminatory capacity already demonstrated by our vector as well as incorporate the exquisite discriminatory capacity of the immune system. Our experience to date with the vaccinia viral vectors has suggested that viral-induced oncolysis alone does not drive an efficient antitumor immune response. This is not surprising when one considers the biology of vaccinia virus. As outlined above, vaccinia has evolved very efficient mechanisms to slow the early development of a Th1 response. Thus, it is not surprising that the wild-type vaccinia virus is not able to efficiently cross-prime the immune system to tumor antigens. We hypothesize that the best way to reverse this process and create an effective in vivo tumor oncolysis vaccine would be through expression of a cytokine capable of driving a Th1/Tc1 immune response. However, as previously mentioned, one of the chief limitations to efficient in vivo viral replication and high tumor infection rates is premature immune clearance of the virus. Insertion of cytokines would be expected to exacerbate this problem. Our preliminary observations have indicated that insertion of FAS - L into our vector paradoxically decreases its efficacy in immunocompetent animals, presumably by promoting clearance of the virus before a significant percentage of tumor infection has occurred (unpublished observations). This decrease in viral replication efficiency has been observed in other recombinant vaccinia systems using cytokine transgenes. Perera et $\mathrm{al}^{49}$ have demonstrated that recombinant vaccinia containing IL-2 or IL-15 demonstrates markedly reduced replication efficacy in vivo. Similarly, others have shown that recombinant vaccinia virus expression of a variety of cytokines results in marked impairment of in vivo replication efficiency. ${ }^{50-52}$ However, whereas it is clear that premature clearance of the virus is harmful to in vivo viral replication and consequently to the direct oncolytic properties of the virus, it is not as clear that it will diminish the immunostimulatory/vaccination capabilities. ${ }^{53}$ Gherardi et al, using a recombinant VV expressing both the $I L-12$ gene and the HIV-env gene, showed that expression of IL-12 in the replication-competent vector directly decreased the in vivo viral replication efficiency. However, when lower titers of virus were used to inoculate the animal, IL-12 transgene expression resulted in augmentation of the anti-HIV-ENV response. ${ }^{54}$ Only empirical testing will tell us whether the enhanced bystander capabilities of a particular cytokine recombinant vaccinia will outweigh the diminution of its direct oncolytic effect. However, creation of an inducible transgene expression system for vaccinia that would allow for the coordinated expression of the immunostimulatory cytokine gene, only after significant in vivo tumor infection had occurred, would theoretically alleviate this concern.

\section{Clinical experience to date}

Whereas no clinical trials to date have directly studied vaccinia virus as an oncolytic therapy, there have been numerous trials utilizing a less efficient strain of replicating vaccinia as a vaccine for the treatment of tumors as well as treatment of infectious diseases such as HIV. Replicating vaccinia virus has been delivered as subcutaneous, intramuscular, intratumoral, and intravesical injections in clinical immunotherapy trials without significant side effects. ${ }^{55-58}$ Doses of up to $10^{9}$ pfu have been delivered without significant toxicity. The most relevant data come from a trial reported by Mastrangelo et al where up to $2 \times 10^{7}$ pfu of replicating $\mathrm{NYCBH}$ strain vaccinia expressing GMCSF was directly injected into melanoma deposits. Of seven patients treated, three patients had mixed responses with regression of treated and untreated dermal metastases, one patient had a partial response with regression of injected and uninjected regional dermal metastases, and one patient with only dermal metastases confined to the scalp achieved a complete remission. It is not clear how much of the antitumor effect was secondary to viral replication, but there is hope that with a more efficient replicating virus, large tumors can be eradicated. The general trend in immunotherapy trials has been to move away from replicating poxviruses, and utilize nonreplicating variants and species such as MVA and fowlpox. Our goal has been to take advantage of the profound efficiency of WR strain vaccinia to enhance the direct tumor cell killing, and increase the percent of cells within a tumor expressing a therapeutic transgene.

\section{Conclusion}

We have focused on the development of a replicationcompetent vaccinia vector derived from the WR strain for the treatment of human cancers. Through selective deletion of important genes, we have developed a highly tumorselective strain that retains its ability to infect and destroy tumor cells while demonstrating little to no pathogenicity in normal tissues. Like many other replication-competent viral 
vectors currently being investigated for the treatment of cancer, the clinical utility of this virus is limited by premature immunologic clearance of the virus and inadequate bystander effects. We are actively investigating strategies to circumvent these issues, including transient immunosuppression, creation of inducible transgene expression systems, and insertion of immunostimulatory cytokines to generate in vivo tumor oncolysates that will serve as a vaccine. Compared to other replicating vectors such as herpes and adenovirus, the study of vaccinia virus is in its infancy. We believe that with time and more experience with this vector, some of its unique advantages will prove superior to other viral vectors.

\section{References}

1. Fenner F. In: Fields BN, Knipe DM, Howley PM, eds. Fields Virology. Lippincott-Raven: Philadelphia; 1996: 2673-2700.

2. Fentiman IS, Rubens RD, Hayward JI. A comparison of intracavitary talcum and tetracycline for the control of pleural effusion secondary to breast cancer. Eur J Cancer Clin Oncol. 1986;22:1079.

3. Fenner F, Wittek R, Dumbell KR. The Orthopoxviruses. Academic Press: New York; 1989:143-170.

4. Antoine G, Scheiflinger F, Dorner F, Falkner FG. The complete genomic sequence of the modified vaccinia Ankara strain: comparison with other orthopoxviruses. Virology. 1998;242: 365-396.

5. Goebel SJ, Johnson GP, Perkus ME, Davis SW, Winslow JP, Paoletti E. The complete DNA sequence of vaccinia virus. Virology. 1990;179:247-266.

6. Hsiao JC, Chung CS, Chang W. Vaccinia virus envelope D8L protein binds to cell surface chondroitin sulfate and mediates the adsorption of intracellular mature virions to cells. $J$ Virol. 1999; 73:8750-8761.

7. Chung CS, Hsiao JC, Chang YS, Chang W. A27L protein mediates vaccinia virus interaction with cell surface heparan sulfate. J Virol. 1998;72:1577-1585.

8. Moss B, Earl PL. Expression of proteins in mammalian cells using vaccinia viral vectors. Curr Prot Mol Biol. 1998;43:16.

9. McIntosh AAG, Smith GL. Vaccinia virus glycoprotein A34R is required for infectivity of extracellular enveloped virus. J Virol. 1996;70:272-281.

10. Seet BT, McFadden G. Viral chemokine-binding proteins. $J$ Leukoc Biol. 2002;72:24-34.

11. Alcami A, Symons JA, Collins PD, Williams TJ, Smith GL. Blockade of chemokine activity by a soluble chemokine binding protein from vaccinia virus. J Immunol. 1998;160:624-633.

12. Mahalingam S, Karupiah G. Modulation of chemokines by poxvirus infections. Curr Opin Immunol. 2000;12:409-412.

13. Symons JA, Alcami A, Smith GL. Vaccinia virus encodes a soluble type I interferon receptor of novel structure and broad species specificity. Cell. 1995;81:551-560.

14. Najarro P, Traktman P, Lewis JA. Vaccinia virus blocks gamma interferon signal transduction: viral VH1 phosphatase reverses Stat1 activation. $J$ Virol. 2001;75:3185-3196.

15. Smith VP, Bryant NA, Alcami A. Ectromelia, vaccinia and cowpox viruses encode secreted interleukin-18-binding proteins. J Gen Virol. 2000;81:1223-1230.

16. Calderara S, Xiang Y, Moss B. Orthopoxvirus IL-18 binding proteins: affinities and antagonist activities. Virology. 2001;279: $22-26$.

17. Novick D, Kim SH, Fantuzzi G, Reznikov LL, Dinarello CA,
Rubinstein M. Interleukin-18 binding protein: a novel modulator of the Th1 cytokine response. Immunity. 1999;10:127136.

18. Engelstad M, Howard ST, Smith GL. A constitutively expressed vaccinia gene encodes a $42-\mathrm{kDa}$ glycoprotein related to complement control factors that forms part of the extracellular virus envelope. Virology. 1992;188:801-810.

19. Bowie A, Kiss-Toth E, Symons JA, Smith GL, Dower SK, O'Neill LA. A46R and A52R from vaccinia virus are antagonists of host IL -1 and toll-like receptor signaling. Proc Natl Acad Sci USA. 2000;97:10162-10167.

20. Howard ST, Chan YS, Smith GL. Vaccinia virus homologues of the Shope fibroma virus inverted terminal repeat proteins and a discontinuous ORF related to the tumor necrosis factor receptor family. Virology. 1991;180:633-647.

21. Smith GL, Howard ST, Chan YS. Vaccinia virus encodes a family of genes with homology to serine proteinase inhibitors. J Gen Virol. 1989;70:2333-2343.

22. Sutter G, Moss B. Novel vaccinia vector derived from the host range restricted and highly attenuated MVA strain of vaccinia virus. Dev Biol Stand. 1995;84:195-200.

23. Blanchard TJ, Alcami A, Andrea P, Smith GL. Modified vaccinia virus Ankara undergoes limited replication in human cells and lacks several immunomodulatory proteins: implications for use as a human vaccine. J Gen Virol. 1998;79:1159-1167.

24. Deonarain R, Alcami A, Alexiou M, Dallman MJ, Gewert DR, Porter AC. Impaired antiviral response and alpha/beta interferon induction in mice lacking beta interferon. $J$ Virol. 2000;74:3404-3409.

25. van Den Broek M, Bachmann MF, Kohler G, Barner M, Escher R, Zinkernagel R, Kopf M. IL - 4 and IL - 10 antagonize IL - 12mediated protection against acute vaccinia virus infection with a limited role of IFN-gamma and nitric oxide synthetase 2 . J Immunol. 2000;164:371-378.

26. Whitman ED, Tsung K, Paxson J, Norton JA. In vitro and in vivo kinetics of recombinant vaccinia virus cancer-gene therapy. Surgery. 1994;116:183-188.

27. Moss B, Carroll MW, Wyatt LS, et al. Host range restricted, non-replicating vaccinia virus vectors as vaccinia candidates, in Design and Production of Vaccines. New York: Plenum Press; 1996:7-13.

28. Ricketts TF. Diagnosis of Smallpox. London: Cassell and Company; 1966:6-13.

29. Neeman M, Abramovitch R, Schiffenbauer YS, Tempel C. Regulation of angiogenesis by hypoxic stress: from solid tumours to the ovarian follicle. Int J Exp Pathol. 1997;78:5770.

30. McCart JA, Hu YK, Alexander HR, Libutti SK, Moss B, Bartlett DL. A combined thymidine kinase/vaccinia growth factor deleted vaccinia virus as a vector for cancer gene therapy. Proc Am Soc Gene Ther. 1999;2:160.

31. Puhlmann M, Brown CK, Gnant M, et al. Vaccinia as a vector for tumor directed gene therapy: biodistribution of a thymidine kinase deleted mutant. Cancer Gene Ther. 2000;7:66-73.

32. Gnant MF, Noli LA, Irvine KR, et al. Tumor specific gene delivery using recombinant vaccinia virus in a rabbit model of unresectable liver metastases - pattern of gene expression, vector elimination and immune response. J Natl Cancer Inst. 1999;91:1744-1750.

33. McCart JA, Puhlmann M, Lee J, et al. Complex interactions between the replicating oncolytic effect and the enzyme/ prodrug effect of vaccinia-mediated tumor regression. Gene Ther. 2000;7:1217-1223.

34. Gnant MFX, Puhlmann M, Alexander HR Jr, Bartlett DL. Systemic administration of a recombinant vaccinia virus expressing the cytosine deaminase gene and subsequent 
treatment with 5 -fluorocytosine leads to tumor specific gene expression and prolongation of survival in mice. Cancer Res. 1999;59:3396-3404.

35. Puhlmann M, Gnant M, Brown CK, Alexander HR, Bartlett DL. Thymidine kinase deleted vaccinia virus expressing purine nucleoside phosphorylase as a vector for tumor directed gene therapy. Hum Gene Ther. 1999;10:649-657.

36. McCart JA, Ward JM, Lee J, et al. Systemic cancer therapy with a tumor selective vaccinia virus mutant lacking thymidine kinase and vaccinia growth factor genes. Cancer Res. 2001;61:8751-8757, 12-15 (Reference Type: Generic).

37. Buller RML, Chakrabarti S, Cooper JA, Twardzik DR, Moss B. Deletion of the vaccinia virus growth factor gene reduces virus virulence. J Virol. 1988;62:866-874.

38. Bischoff JR, Kim DH, Williams A, et al. An adenovirus mutant that replicates selectively in p53-deficient human tumor cells [see comments]. Science. 1996;274:373-376.

39. Ray C, Black RA, Kronheim SR, et al. Viral inhibition of inflammation: cowpox virus encodes an inhibitor of the interleukin-1 $\alpha$ converting enzyme. Cell. 1992;69:597-604.

40. Kettle S, Blake NW, Law KM, Smith GL. Vaccinia virus serpins B13R (SPI-2) and B22R (SPI-1) encode $M_{\mathrm{r}} 385$ and $40 \mathrm{~K}$, intracellular polypeptides that do not affect virus virulence in a murine intranasal model. Virology. 1995;206:136147.

41. Shisler JL, Isaacs SN, Moss B. Vaccinia virus serpin - 1 deletion mutant exhibits a host range defect characterized by low levels of intermediate and late mRNAs. Virology. 1999;262:298-311.

42. Perkus ME, Goebel SJ, Davis SW, et al. Vaccinia virus host range genes. Virology. 1990;179:276-286.

43. Chung CS, Vasilevskaya IA, Wang SC, Bair CH, Chang W. Apoptosis and host restriction of vaccinia virus in RK13 cells. Virus Res. 1997;52:121-132.

44. Oguiura N, Spehner D, Drillien R. Detection of a protein encoded by the vaccinia virus $\mathrm{C} 7 \mathrm{~L}$ open reading frame and study of its effect on virus multiplication in different cell lines. J Gen Virol. 1993;74:1409-1413.

45. Ramsey-Ewing AL, Moss B. Complementation of a vaccinia virus host-range $K 1 L$ gene deletion by the nonhomologous CP77 gene. Virology. 1996;222:75-86.

46. Galmiche MC, Rindisbacher L, Wels W, Wittek R, Buchegger F. Expression of a functional single chain antibody on the surface of extracellular enveloped vaccinia virus as a step towards selective tumour cell targeting. J Gen Virol. 1997;78: 3019-3027.
47. Hu Y, Lee J, McCart JA, et al. Yaba like disease virus: an alternating replicating pox vector for cancer gene therapy. J Virol. 2001;75:10300-10308.

48. Sharma DP, Ramsay AJ, Maguire DJ, Rolph MS, Ramshaw IA. Interleukin-4 mediates down regulation of antiviral cytokine expression and cytotoxic T-lymphocyte responses and exacerbates vaccinia virus infection in vivo. J Virol. 1996;70:71037107.

49. Perera LP, Goldman CK, Waldmann TA. Comparative assessment of virulence of recombinant vaccinia viruses expressing IL-2 and IL-15 in immunodeficient mice. Proc Natl Acad Sci USA. 2001;98:5146-5151.

50. Karupiah G, Woodhams CE, Blanden RV, Ramshaw IA. Immunobiology of infection with recombinant vaccinia virus encoding murine IL - 2. Mechanisms of rapid viral clearance in immunocompetent mice. J Immunol. 1991;147:4327-4332.

51. Ruby J, Bluethmann H, Aguet M, Ramshaw IA. CD40 ligand has potent antiviral activity. Nat Med. 1995;1:437-441.

52. Sambhi SK, Kohonen-Corish MR, Ramshaw IA. Local production of tumor necrosis factor encoded by recombinant vaccinia virus is effective in controlling viral replication in vivo. Proc Natl Acad Sci USA. 1991;88:4025-4029.

53. Ramirez JC, Gherardi MM, Rodriguez D, Esteban M. Attenuated modified vaccinia virus Ankara can be used as an immunizing agent under conditions of preexisting immunity to the vector. J Virol. 2000;74:7651-7655.

54. Gherardi MM, Ramirez JC, Rodriguez D, et al. IL-12 delivery from recombinant vaccinia virus attenuates the vector and enhances the cellular immune response against HIV-1 Env in a dose-dependent manner. J Immunol. 1999;162:6724-6733.

55. Lattime EC, Lee SS, Eisenlohr LC, Mastrangelo MJ. In situ cytokine gene transfection using vaccinia virus vectors. Semin Oncol. 1996;23:88-100.

56. Mastrangelo MJ, Maguire HC Jr, Eisenlohr LC, et al. Intratumoral recombinant GM-CSF-encoding virus as gene therapy in patients with cutaneous melanoma. Cancer Gene Ther. 1998;6:409-422.

57. Overwijk W, Lee DS, Surman DR, et al. Vaccination with a recombinant vaccinia virus encoding a "self" antigen induces autoimmune vitiligo and tumor cell destruction in mice: requirement for CD4 ${ }^{+}$T lymphocytes. Proc Natl Acad Sci. 1999;96:2982-2987.

58. Rosenberg SA, Blaese RM, Brenner MK, et al. Human gene marker/therapy clinical protocols. Hum Gene Ther. 1999;10: 3067-3123. 